\title{
Automated cortical projection of EEG sensors: Anatomical correlation via the international $10-10$ system
}

\author{
L. Koessler ${ }^{\text {a,b }}$, L. Maillard ${ }^{\text {b }}$, A. Benhadid ${ }^{\text {a }}$, J.P. Vignal ${ }^{\text {b }}$, J. Felblinger ${ }^{\text {a }}$, H. Vespignani ${ }^{\text {b }}$, M. Braun ${ }^{\text {a,c,d,* }}$, \\ a INSERM U947, Nancy University, France \\ ${ }^{\mathrm{b}}$ Neurology Department, University Hospital, Nancy, France \\ c Neuroradiology Department, University Hospital, Nancy, France \\ d Anatomy Department, Nancy University, France
}

\section{A R T I C L E I N F O}

\section{Article history:}

Received 10 July 2008

Revised 31 December 2008

Accepted 5 February 2009

Available online 20 February 2009

\section{Keywords:}

Human brain mapping

International 10-10 system

Cranio-cerebral correlation

Trans-magnetic stimulation

Near-infrared spectroscopy

Electroencephalography

\begin{abstract}
A B S T R A C T
Several studies have described cranio-cerebral correlations in accordance with the 10-20 electrode placement system. These studies have made a significant contribution to human brain imaging techniques, such as near-infrared spectroscopy and trans-magnetic stimulation. With the recent development of high resolution EEG, an extension of the 10-20 system has been proposed. This new configuration, namely the 1010 system, allows the placement of a high number (64-256) of EEG electrodes. Here, we describe the craniocerebral correlations with the 10-10 system. Thanks to the development of a new EEG-MRI sensor and an automated algorithm which enables the projection of electrode positions onto the cortical surface, we studied the cortical projections in 16 healthy subjects using the Talairach stereotactic system and estimated the variability of cortical projections in a statistical way. We found that the cortical projections of the 10-10 system could be estimated with a grand standard deviation of $4.6 \mathrm{~mm}$ in $x, 7.1 \mathrm{~mm}$ in $y$ and $7.8 \mathrm{~mm}$ in $z$. We demonstrated that the variability of projections is greatest in the central region and parietal lobe and least in the frontal and temporal lobes. Knowledge of cranio-cerebral correlations with the 10-10 system should enable to increase the precision of surface brain imaging and should help electrophysiological analyses, such as localization of superficial focal cortical generators.
\end{abstract}

(c) 2009 Elsevier Inc. All rights reserved.

\section{Introduction}

Localization of brain generators in a non-invasive way has been improved with the development of high resolution EEG. This technique allows the acquisition of EEG signals with a high spatial resolution at the level of the scalp, and high temporal definition. Manufacturers of EEG supplies have recognized this, and electrode caps which enable easy placement of electrodes according to the 1010 standard are available. Currently, more and more researchers are moving to an even higher number of channels and EEG acquisition systems with 128 channels are no longer uncommon. Several authors have shown that a high number of electrodes increases the precision of the localization of intra-cerebral generators which are at the origin of the surface signals (Lantz et al., 2003; Michel et al., 2004).

The positioning of EEG electrodes is defined according to external landmarks such as nasion, inion and pre-auriculars, but also according to the cerebral structures beneath each sensor (Jasper, 1958). A fundamental assumption of the system is that there is a reliable correlation between scalp sensor location and the underlying cerebral

\footnotetext{
* Corresponding author. INSERM ERI13, Nancy University, France. Fax: + 33383852236 E-mail address: m.braun@chu-nancy.fr (M. Braun).
}

structure. Some studies have examined the validity of the structural correlation using cadavers (Blume et al., 1974; Jasper, 1958), X-rays (Morris et al., 1986), CT scans (Homan et al., 1987; Myslobodsky and Bar-Ziv, 1989; Myslobodsky et al., 1990) and MRI (Steinmetz et al., 1989; Jack et al., 1990; Lagerlund et al., 1993; Towle et al., 1993). An initial study (Homan et al., 1987) plotted the $10-20$ positions on Brodmann's cortical map from the temporal view. These authors successfully described the cranio-cerebral structural relationships on Brodmann's plane. However, no statistical analysis was performed and their methods could not avoid a resolution gap because of the dimensional conversion from space to plane. These problems were overcome by fitting cranial and cerebral surfaces to a sphere (Lagerlund et al., 1993; Towle et al., 1993). These authors described the cortical locations that lie beneath the 10-20 electrodes (cortical projection points) as 3D coordinates. More recently, Okamoto et al. examined the cranio-cerebral correspondences for 17 healthy adults and normalized the 10-20 cortical projection points of the subjects to the standard Montreal Neurological Institute (MNI) and Talairach stereotactic coordinates (reviewed in Brett et al., 2002). Statistical analysis was performed in order to obtain their probabilistic distribution. Automated methods have also been proposed to project head-surface locations onto the cortical surface in structural images (Okamoto and Dan, 2005). 
Knowledge of cortical projections of the 10-10 system has several applications in surface brain imaging such as trans-magnetic stimulation (TMS) or near-infrared spectroscopic (NIRS) imaging (Okamoto et al., 2004). These techniques use indeed the international systems of sensor positioning initially described for EEG. At the opposite of electroencephalography or magnetoencephelography which now uses source imaging techniques for localization purpose (Michel et al., 2004), TMS and NIRS, which only concern the superficial cortical surface, strongly rely on cranio-cerebral correlations.

Although numerous studies exist, the cortical projections of electrodes within the 10-10 system and their anatomical variability have never been described. The purpose of our study was therefore to examine the cranio-cerebral correlations by determining the 10-10 cortical projection points in 16 healthy subjects, describing their locations in Talairach space with statistical estimation of variability and corresponding macro-anatomical and cytoarchitectonic features.

This work could provide a reliable database of cranio-cerebral correlations to researchers and neurophysiologists when they have no structural images for their subjects.

\section{Methods}

\section{Subjects}

Sixteen healthy subjects (six women and 10 men, aged 20 42 years) participated in this study. All subjects were investigated in order to confirm the absence of neurological abnormalities, and all gave their informed consent. The study was approved by the ethics committee (CCPPRB) of our institution.

\section{0-10 Sensor placement}

To reproduce a high-resolution EEG situation, 64 EEG-MRI sensors were taped onto the subject's head (Fig. 1). The EEG-MRI sensors were made by combining an EEG electrode, plastic support and MRI marker (Koessler et al., 2008).

The positioning of the sensors on the subject's head was carried out by four different experienced technologists in accordance with the 10-10 system defined in Oostenveld and Praamstra (2001). The placement of electrodes was based on anatomical landmarks: nasion (Nz), inion (Iz), and left and right pre-auricular points (LPA and RPA). All distances (Nz-Iz, LPA-RPA) and the circumference of the head were measured in order to place the sensors accurately. This extended 10-20 system of electrode placement, also known as the 10-10 system, has been accepted and is currently endorsed as the standard of the American Electroencephalographic Society (Klem et al., 1999) and the International Federation of Societies for Electroencephalography and Clinical Neurophysiology (Nuwer et al., 1998).

\section{Magnetic resonance imaging}

All MRI examinations were performed on a 1.5 T GE Signa (GE Healthcare, Milwaukee, WI) with an eight element coil. Special care was taken during the examination to avoid movement of the scalp or any of the sensors. The parameters of the MR sequence were selected to both accurately detect the EEG sensors and produce precise anatomical brain images. In addition, the complete MR procedure was performed fast enough to avoid discomfort to the subject and to reduce artefacts caused by movement. To do this, we used a 3D spoiled gradient echo sequence ( $\mathrm{TR}=20 \mathrm{~ms}, \mathrm{TE}=3 \mathrm{~ms}, \alpha=35^{\circ}$ ) with a $23 \mathrm{~cm}$ field of view, $192 * 192$ matrix and 200 slices. Slice thickness was $1.2 \mathrm{~mm}$, without any gap between slices. A large bandwidth $(31.2 \mathrm{kHz})$ was used to reduce distortion due to magnetic susceptibility.

\section{Determination of $10-10$ sensor positions}

For each subject, the EEG-MRI sensors were detected on the MR images. To determine the 10-10 sensor positions, we used the ALLES (automatic localization and labelling of the EEG sensor) method to detect and label the EEG sensors (Koessler et al., 2008). First, an automated thresholding based on the histogram of the MR volume was performed to separate the marker from the rest of the volume (head and background). Then, a Delaunay convex hull was built to detect the 3D coordinates of the sensors in an automated way. Finally, the estimated locations of the EEG electrodes were projected onto an ellipsoid that modelled the patient's head. Each sensor was then labelled as a function of its coordinates. A visual inspection of the MR images was performed by an experienced neurophysiologist to avoid and manually correct dots considered incorrectly as sensors (false positives) and sensors not detected (false negatives). Finally, the algorithm wrote the list of estimated EEG sensor coordinates in a file to be used with source localization software.

\section{Projection of EEG sensors on the cortical surface}

To perform this task an additional function was inserted in the ALLES algorithm (Fig. 2). For all subjects, we modelled the head tissues (brain, skull and scalp) in a realistic way. The models were based on a segmentation which used MR intensity values. Boundary element model, which describes the individual surfaces by triangulation, was about 1700-2000 nodes per model. The segmentation process and identification of the three compartments of isoconductivity were performed with Advance Source Analysis software (ANT, Enschede, Netherlands). All head models were generated by the same experienced operator. In our study, more attention was paid to the brain compartments. Brain meshes were defined using the smallest triangle possible (sides about $1 \mathrm{~mm}$ ) and with a weak smoothing filter.

Finally, meshes that contained vertices and polygons were produced by ASA software. For each subject, the brain mesh was introduced into

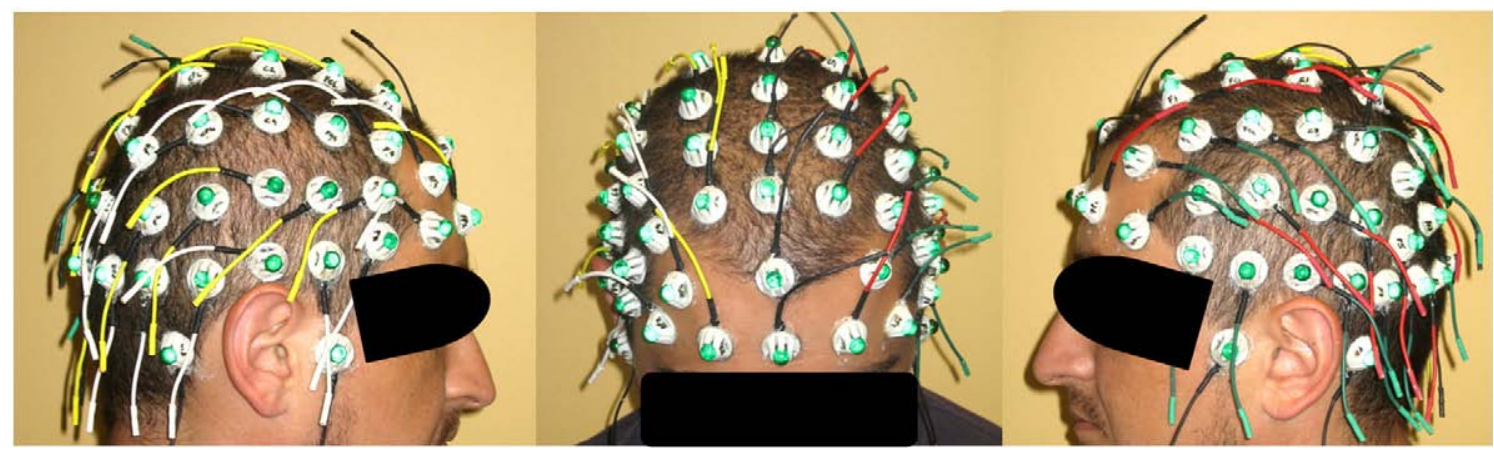

Fig. 1. Right side, front side and left side of the subject's head with 64 EEG-MRI sensors. 


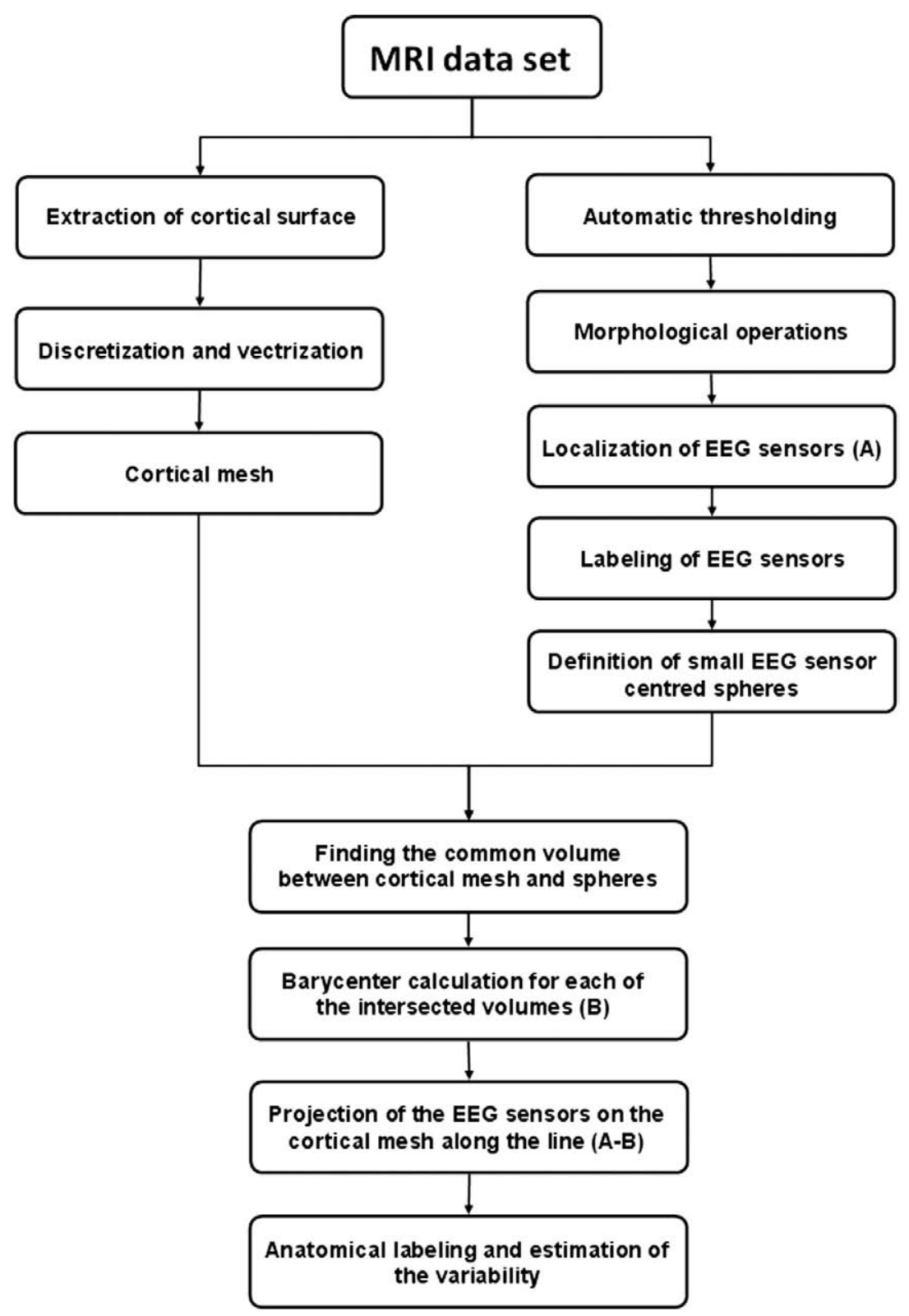

Fig. 2. Flow chart showing the algorithm used in this study.

the ALLES algorithm. A sphere with an arbitrary radius $(1 \mathrm{~cm})$ was then centred at each EEG sensor coordinate $(S)$ detected automatically by ALLES. For each of the EEG sensors, we looked for the cortical vertices that were within the predetermined radius of the EEG ball. That is, we selected all cortical vertices that were at a distance $d$ from the EEG sensor less than the radius of the EEG sphere (i.e. the identified vertices belonged to the intersected volume between the cortical mesh and the EEG virtual sphere). For each intersected volume, we looked for the unique barycentre $(G)$. Finally, EEG sensor coordinates were projected onto the cortical mesh $(C)$ using the intersection point between the line $G-S$ and the cortical surface (Fig. 3). A list of cortical positions defined in the fiducial system was finally written by the ALLES algorithm. 3D visualization of the cortical mesh and cortical projection dots was possible using the graphic user interface.

\section{Labelling and statistical analysis of cortical points}

Labelling of cortical points requires the definition of a common spatial reference. For each subject, we transformed original coordi- nate system to Talairach system. At the origin, all images were in the original MRI system. In this MRI system, the $x$ axis points forward, the $y$ axis to the left, and the $z$ axis upward. Origin of this system is the right inferior posterior corner of the MRI block. Using ASA, we first determined three anatomical landmarks (nasion, left and right pre-auricular points) in order to define the fiducial system which is also used for head modelling and sensor localization. In this fiducial system, the $y$ direction is determined by the connecting line between the two pre-auricular points (pointing left). The origin is obtained by projecting the nasion orthogonally onto this line. The $x$ axis points from the origin to the nasion and the $z$ axis is perpendicular on the plan defined by $x$ and $y$ axes. Then, we defined several cortical points in the MR volume. First, we determined the anterior commissure (AC) and posterior commissure (PC). The AC-PC line determines the orientation of the $y$ axis, which points in the anterior direction. The $z$ axis is orientated upward and perpendicular to the AC-PC line between the two hemispheres. It lies in a plane defined by the AC-PC line and the interhemispherical point, which we chose at an arbitrary spot in the interhemispherical fissure. The $x$ axis points to the left 


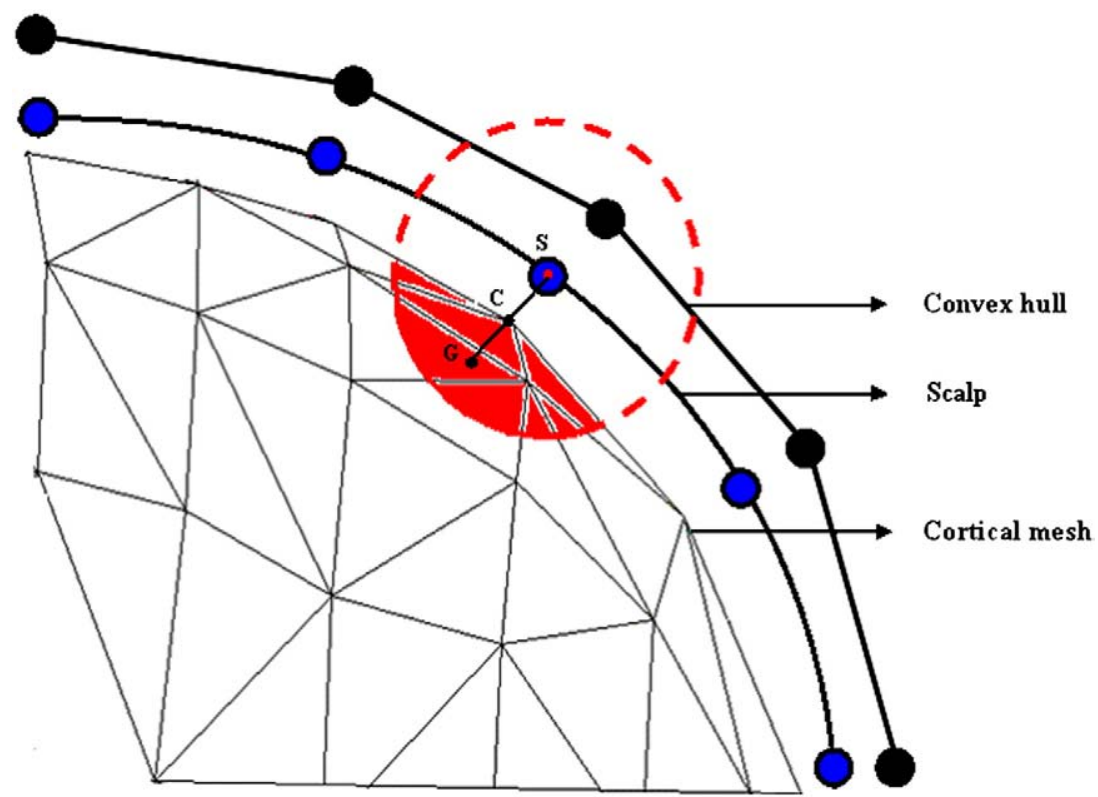

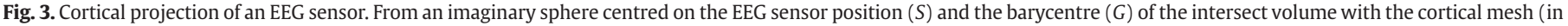
red), cortical position was located along the line $G-S$.

side, orthogonal to the other two axes. Second, six other cortical points were defined to introduce the Talairach system, which is a piecewise linear transformation of the AC-PC system: anterior and posterior point (AP and PP; i.e. point of the cortex with maximum and minimum $x$ coordinates), superior and inferior points (SP and IP; i.e. point of the cortex with maximum and minimum $z$ coordinates), and right and left points (RP and LP; i.e. point of the cortex with maximum and minimum $y$ coordinates). When all anatomical points were placed in order to implement the Talairach system, ASA software changed in an automated way the origin of the volume which was first defined in the fiducial system (see Appendix A). All these mathematical transformations preserved the anatomical information about each subject since there was no spatial smoothing and normalization. Each subject's own space was transformed to the corresponding Talairach system (Fig. 4).

All sets of 10-10 cortical points were labelled using the Talairach Daemon programme (Lancaster et al., 2000). Visual inspections by two senior anatomists were also carried out for each sensor in order to confirm the precise labelling of Talairach Daemon and to avoid equivocal projections. We then averaged all cortical coordinates in order to obtain mean cortical coordinates and standard deviations for our population:

$$
(\bar{x}, \bar{y}, \bar{z})=\left(\frac{\sum x}{n}, \frac{\sum y}{n}, \frac{\sum z}{n}\right)\left|\mathrm{SD}=\sqrt{\frac{\sum(x-\bar{x})^{2}+\sum(y-\bar{y})^{2}+\sum(z-\bar{z})^{2}}{n-1}}\right| .
$$

Each mean 10-10 cortical coordinate was then labelled with the Talairach Daemon programme. Then, in the population, we estimated the likely Brodmann area (BA) beneath each sensor: for each sensor, we presented the corresponding Brodmann areas and their frequency in the population. This frequency was expressed as a percentage across all the subjects. For example, if a sensor projected to BA 10 in 12 subjects, and to BA 46 in 4, results were displayed as BA 10 (75\%) and BA 46 (25\%). The likely Brodmann area is the one which had the biggest percentage i.e. the one which was the most frequently found in the population. The same method was used to estimate the macroanatomical (sulcal and gyral) variability.

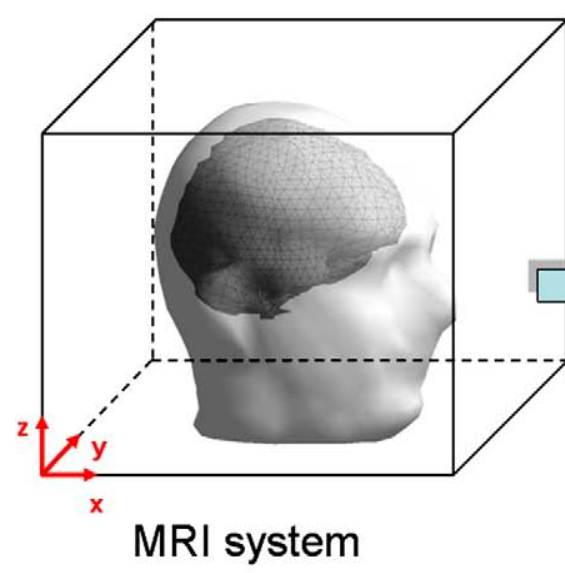

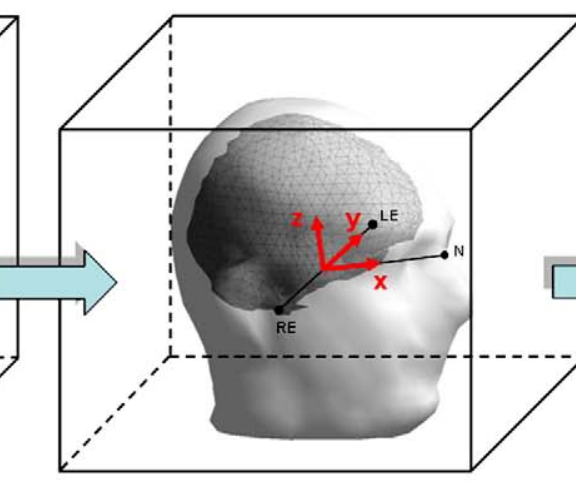

Fiducial system

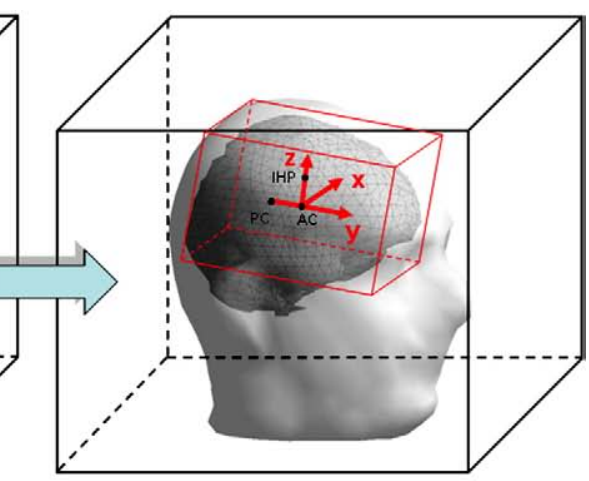

Talairach system

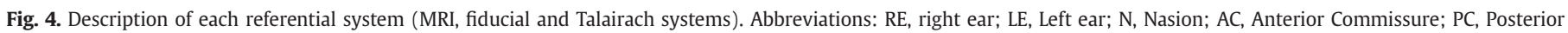
Commissure; IHP, Interhemispherical point. 


\section{Anatomical visualization}

Each mean cortical coordinate was introduced into the programme of the International Neuroimaging Consortium (INC, http://www. neurovia.umn.edu) in order to create a macro-anatomical atlas of
10-10 cortical positions. For each sensor, we obtained the projected dot in axial, sagittal and coronal views. Moreover, each dot was reported in the stereotactic atlas of Talairach. A mean MRI was created from the MRIs of the 16 subjects. This step was facilitated using the same common reference. We then segmented the mean MR volume

Table 1

Anatomical locations of international $10-10$ cortical projections

\begin{tabular}{|c|c|c|c|c|c|c|}
\hline \multirow[t]{2}{*}{ Labels } & \multicolumn{3}{|c|}{ Talairach coordinates } & \multirow[t]{2}{*}{ Gyri } & & \multirow[t]{2}{*}{$\mathrm{BA}$} \\
\hline & $x \operatorname{avg}(\mathrm{mm})$ & $y \operatorname{avg}(\mathrm{mm})$ & $z \operatorname{avg}(\mathrm{mm})$ & & & \\
\hline$\overline{\text { FP1 }}$ & $-21.2 \pm 4.7$ & $66.9 \pm 3.8$ & $12.1 \pm 6.6$ & L FL & Superior frontal G & 10 \\
\hline $\mathrm{FPz}$ & $1.4 \pm 2.9$ & $65.1 \pm 5.6$ & $11.3 \pm 6.8$ & M FL & Bilat. medial & 10 \\
\hline FP2 & $24.3 \pm 3.2$ & $66.3 \pm 3.5$ & $12.5 \pm 6.1$ & R FL & Superior frontal G & 10 \\
\hline AF7 & $-41.7 \pm 4.5$ & $52.8 \pm 5.4$ & $11.3 \pm 6.8$ & L FL & Middle frontal G & 10 \\
\hline AF3 & $-32.7 \pm 4.9$ & $48.4 \pm 6.7$ & $32.8 \pm 6.4$ & L FL & Superior frontal G & 9 \\
\hline $\mathrm{AFz}$ & $1.8 \pm 3.8$ & $54.8 \pm 7.3$ & $37.9 \pm 8.6$ & M FL & Bilat. medial & 9 \\
\hline AF4 & $35.1 \pm 3.9$ & $50.1 \pm 5.3$ & $31.1 \pm 7.5$ & L FL & Superior frontal G & 9 \\
\hline AF8 & $43.9 \pm 3.3$ & $52.7 \pm 5.0$ & $9.3 \pm 6.5$ & R FL & Middle frontal G & 10 \\
\hline F7 & $-52.1 \pm 3.0$ & $28.6 \pm 6.4$ & $3.8 \pm 5.6$ & L FL & Inferior frontal G & 45 \\
\hline F5 & $-51.4 \pm 3.8$ & $26.7 \pm 7.2$ & $24.7 \pm 9.4$ & L FL & Middle frontal G & 46 \\
\hline F3 & $-39.7 \pm 5.0$ & $25.3 \pm 7.5$ & $44.7 \pm 7.9$ & L FL & Middle frontal G & 8 \\
\hline $\mathrm{F} 1$ & $-22.1 \pm 6.1$ & $26.8 \pm 7.2$ & $54.9 \pm 6.7$ & L FL & Superior frontal G & 6 \\
\hline $\mathrm{Fz}$ & $0.0 \pm 6.4$ & $26.8 \pm 7.9$ & $60.6 \pm 6.5$ & M FL & Bilat. medial & 6 \\
\hline $\mathrm{F} 2$ & $23.6 \pm 5.0$ & $28.2 \pm 7.4$ & $55.6 \pm 6.2$ & R FL & Superior frontal G & 6 \\
\hline F4 & $41.9 \pm 4.8$ & $27.5 \pm 7.3$ & $43.9 \pm 7.6$ & R FL & Middle frontal G & 8 \\
\hline F6 & $52.9 \pm 3.6$ & $28.7 \pm 7.2$ & $25.2 \pm 7.4$ & R FL & Middle frontal G & 46 \\
\hline F8 & $53.2 \pm 2.8$ & $28.4 \pm 6.3$ & $3.1 \pm 6.9$ & R FL & Inferior frontal G & 45 \\
\hline FT9 & $-53.8 \pm 3.3$ & $-2.1 \pm 6.0$ & $-29.1 \pm 6.3$ & L TL & Inferior temporal G & 20 \\
\hline FT7 & $-59.2 \pm 3.1$ & $3.4 \pm 5.6$ & $-2.1 \pm 7.5$ & L TL & Superior temporal G & 22 \\
\hline FC5 & $-59.1 \pm 3.7$ & $3.0 \pm 6.1$ & $26.1 \pm 5.8$ & L FL & Precentral G & 6 \\
\hline FC3 & $-45.5 \pm 5.5$ & $2.4 \pm 8.3$ & $51.3 \pm 6.2$ & L FL & Middle frontal G & 6 \\
\hline FC1 & $-24.7 \pm 5.7$ & $0.3 \pm 8.5$ & $66.4 \pm 4.6$ & L FL & Superior frontal G & 6 \\
\hline $\mathrm{FCz}$ & $1.0 \pm 5.1$ & $1.0 \pm 8.4$ & $72.8 \pm 6.6$ & M FL & Superior frontal G & 6 \\
\hline FC2 & $26.1 \pm 4.9$ & $3.2 \pm 9.0$ & $66.0 \pm 5.6$ & R FL & Superior frontal G & 6 \\
\hline FC4 & $47.5 \pm 4.4$ & $4.6 \pm 7.6$ & $49.7 \pm 6.7$ & R FL & Middle frontal G & 6 \\
\hline FC6 & $60.5 \pm 2.8$ & $4.9 \pm 7.3$ & $25.5 \pm 7.8$ & R FL & Precentral G & 6 \\
\hline FT8 & $60.2 \pm 2.5$ & $4.7 \pm 5.1$ & $-2.8 \pm 6.3$ & L TL & Superior temporal G & 22 \\
\hline FT10 & $55.0 \pm 3.2$ & $-3.6 \pm 5.6$ & $-31.0 \pm 7.9$ & $\mathrm{R} \mathrm{TL}$ & Inferior temporal G & 20 \\
\hline $\mathrm{T} 7$ & $-65.8 \pm 3.3$ & $-17.8 \pm 6.8$ & $-2.9 \pm 6.1$ & L TL & Middle temporal G & 21 \\
\hline $\mathrm{C} 5$ & $-63.6 \pm 3.3$ & $-18.9 \pm 7.8$ & $25.8 \pm 5.8$ & L PL & Postcentral G & 123 \\
\hline $\mathrm{C} 3$ & $-49.1 \pm 5.5$ & $-20.7 \pm 9.1$ & $53.2 \pm 6.1$ & L PL & Postcentral G & 123 \\
\hline $\mathrm{C} 1$ & $-25.1 \pm 5.6$ & $-22.5 \pm 9.2$ & $70.1 \pm 5.3$ & L FL & Precentral G & 4 \\
\hline $\mathrm{Cz}$ & $0.8 \pm 4.9$ & $-21.9 \pm 9.4$ & $77.4 \pm 6.7$ & M FL & Precentral G & 4 \\
\hline $\mathrm{C} 2$ & $26.7 \pm 5.3$ & $-20.9 \pm 9.1$ & $69.5 \pm 5.2$ & R FL & Precentral G & 4 \\
\hline $\mathrm{C} 4$ & $50.3 \pm 4.6$ & $-18.8 \pm 8.3$ & $53.0 \pm 6.4$ & R PL & Postcentral G & 123 \\
\hline $\mathrm{C} 6$ & $65.2 \pm 2.6$ & $-18.0 \pm 7.1$ & $26.4 \pm 6.4$ & R PL & Postcentral G & 123 \\
\hline T8 & $67.4 \pm 2.3$ & $-18.5 \pm 6.9$ & $-3.4 \pm 7.0$ & $\mathrm{R} T \mathrm{~T}$ & Middle temporal G & 21 \\
\hline TP7 & $-63.6 \pm 4.5$ & $-44.7 \pm 7.2$ & $-4.0 \pm 6.6$ & L TL & Middle temporal G & 21 \\
\hline CP5 & $-61.8 \pm 4.7$ & $-46.2 \pm 8.0$ & $22.5 \pm 7.6$ & L PL & Supramarginal G & 40 \\
\hline $\mathrm{CP} 3$ & $-46.9 \pm 5.8$ & $-47.7 \pm 9.3$ & $49.7 \pm 7.7$ & L PL & Inferior parietal G & 40 \\
\hline $\mathrm{CP} 1$ & $-24.0 \pm 6.4$ & $-49.1 \pm 9.9$ & $66.1 \pm 8.0$ & L PL & Postcentral G & 7 \\
\hline $\mathrm{CPz}$ & $0.7 \pm 4.9$ & $-47.9 \pm 9.3$ & $72.6 \pm 7.7$ & M PL & Postcentral G & 7 \\
\hline $\mathrm{CP} 2$ & $25.8 \pm 6.2$ & $-47.1 \pm 9.2$ & $66.0 \pm 7.5$ & R PL & Postcentral G & 7 \\
\hline $\mathrm{CP} 4$ & $49.5 \pm 5.9$ & $-45.5 \pm 7.9$ & $50.7 \pm 7.1$ & R PL & Inferior parietal G & 40 \\
\hline CP6 & $62.9 \pm 3.7$ & $-44.6 \pm 6.8$ & $24.4 \pm 8.4$ & R PL & Supramarginal G & 40 \\
\hline TP8 & $64.6 \pm 3.3$ & $-45.4 \pm 6.6$ & $-3.7 \pm 7.3$ & $\mathrm{R} T \mathrm{~L}$ & Middle temporal G & 21 \\
\hline P9 & $-50.8 \pm 4.7$ & $-51.3 \pm 8.6$ & $-37.7 \pm 8.3$ & L TL & Tonsile & NP \\
\hline P7 & $-55.9 \pm 4.5$ & $-64.8 \pm 5.3$ & $0.0 \pm 9.3$ & L TL & Inferior temporal G & 37 \\
\hline P5 & $-52.7 \pm 5.0$ & $-67.1 \pm 6.8$ & $19.9 \pm 10.4$ & L TL & Middle temporal G & 39 \\
\hline P3 & $-41.4 \pm 5.7$ & $-67.8 \pm 8.4$ & $42.4 \pm 9.5$ & L PL & Precuneus & 19 \\
\hline P1 & $-21.6 \pm 5.8$ & $-71.3 \pm 9.3$ & $52.6 \pm 10.1$ & L PL & Precuneus & 7 \\
\hline $\mathrm{Pz}$ & $0.7 \pm 6.3$ & $-69.3 \pm 8.4$ & $56.9 \pm 9.9$ & M PL & Superior parietal L & 7 \\
\hline P2 & $24.4 \pm 6.3$ & $-69.9 \pm 8.5$ & $53.5 \pm 9.4$ & R PL & Precuneus & 7 \\
\hline P4 & $44.2 \pm 6.5$ & $-65.8 \pm 8.1$ & $42.7 \pm 8.5$ & R PL & Inferior parietal L & 7 \\
\hline P6 & $54.4 \pm 4.3$ & $-65.3 \pm 6.0$ & $20.2 \pm 9.4$ & $\mathrm{R} T L$ & Middle temporal G & 39 \\
\hline P8 & $56.4 \pm 3.7$ & $-64.4 \pm 5.6$ & $0.1 \pm 8.5$ & $\mathrm{R} T L$ & Inferior temporal G & 19 \\
\hline P10 & $51.0 \pm 3.5$ & $-53.9 \pm 8.7$ & $-36.5 \pm 10.0$ & L OL & Tonsile & NP \\
\hline PO7 & $-44.0 \pm 4.7$ & $-81.7 \pm 4.9$ & $1.6 \pm 10.6$ & R OL & Middle occipital G & 18 \\
\hline $\mathrm{PO} 3$ & $-33.3 \pm 6.3$ & $-84.3 \pm 5.7$ & $26.5 \pm 11.4$ & R OL & Superior occipital G & 19 \\
\hline $\mathrm{POz}$ & $0.0 \pm 6.5$ & $-87.9 \pm 6.9$ & $33.5 \pm 11.9$ & $\mathrm{M} \mathrm{OL}$ & Cuneus & 19 \\
\hline $\mathrm{PO} 4$ & $35.2 \pm 6.5$ & $-82.6 \pm 6.4$ & $26.1 \pm 9.7$ & R OL & Superior occipital G & 19 \\
\hline PO8 & $43.3 \pm 4.0$ & $-82.0 \pm 5.5$ & $0.7 \pm 10.7$ & R OL & Middle occipital G & 18 \\
\hline 01 & $-25.8 \pm 6.3$ & $-93.3 \pm 4.6$ & $7.7 \pm 12.3$ & L OL & Middle occipital G & 18 \\
\hline $\mathrm{Oz}$ & $0.3 \pm 5.9$ & $-97.1 \pm 5.2$ & $8.7 \pm 11.6$ & M OL & Cuneus & 18 \\
\hline $\mathrm{O} 2$ & $25.0 \pm 5.7$ & $-95.2 \pm 5.8$ & $6.2 \pm 11.4$ & R OL & Middle occipital G & 18 \\
\hline
\end{tabular}

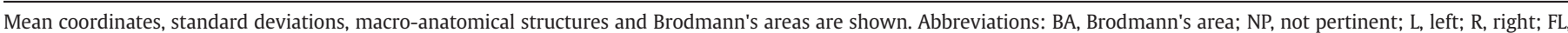
frontal lobe; TL, temporal lobe; PL, parietal lobe; OL, occipital lobe; G, gyrus; L, lobule. 
with ASA software in order to obtain the cortical mesh. Finally, cortical projections were co-registered with the cortical mesh.

\section{Results}

Cortical projections of the 10-10 sensor system

The mean stereotactic coordinates and standard deviations of the locations of the 10-10 cortical projection points based on the 16 subjects expressed in Talairach space are presented in Table 1. Concerning the spatial dispersion around the mean cortical coordinates, we calculated a grand standard deviation of approximately $4.6 \mathrm{~mm}$ in $x, 7.1 \mathrm{~mm}$ in $y$ and $7.8 \mathrm{~mm}$ in $z$. Fig. 5 presents each mean cortical projection onto a Ch2bet brain template with anatomical structures. We calculated for each projection the global standard deviation (i.e. the 3D volume defined by standard deviation in $x, y$ and $z$ ). The diameter of red circles is proportional to the normalized 3D volume of a corresponding sensor. In this work, Fp2 has the smallest global standard deviation of $67 \mathrm{~mm}^{3}$ and P1 the biggest at $548 \mathrm{~mm}^{3}$. We normalized all global standard deviations by assigning a diameter of $1 \mathrm{~mm}$ for Fp2 and a $10 \mathrm{~mm}$ diameter circle for P1. All other global standard deviations were ranged accordingly.

A perfect analogy was observed between macro-anatomical labels and a transverse view (right-left) of the scalp. For example, 01 and 02 sensors were projected on a middle occipital gyrus, T7 and T8 sensors on a middle temporal gyrus, and $\mathrm{C} 1$ and $\mathrm{C} 2$ on the precentral gyrus. Visualization of the projected dots on the mean cortical volume segmented from all MR volumes is depicted in Fig. 6.

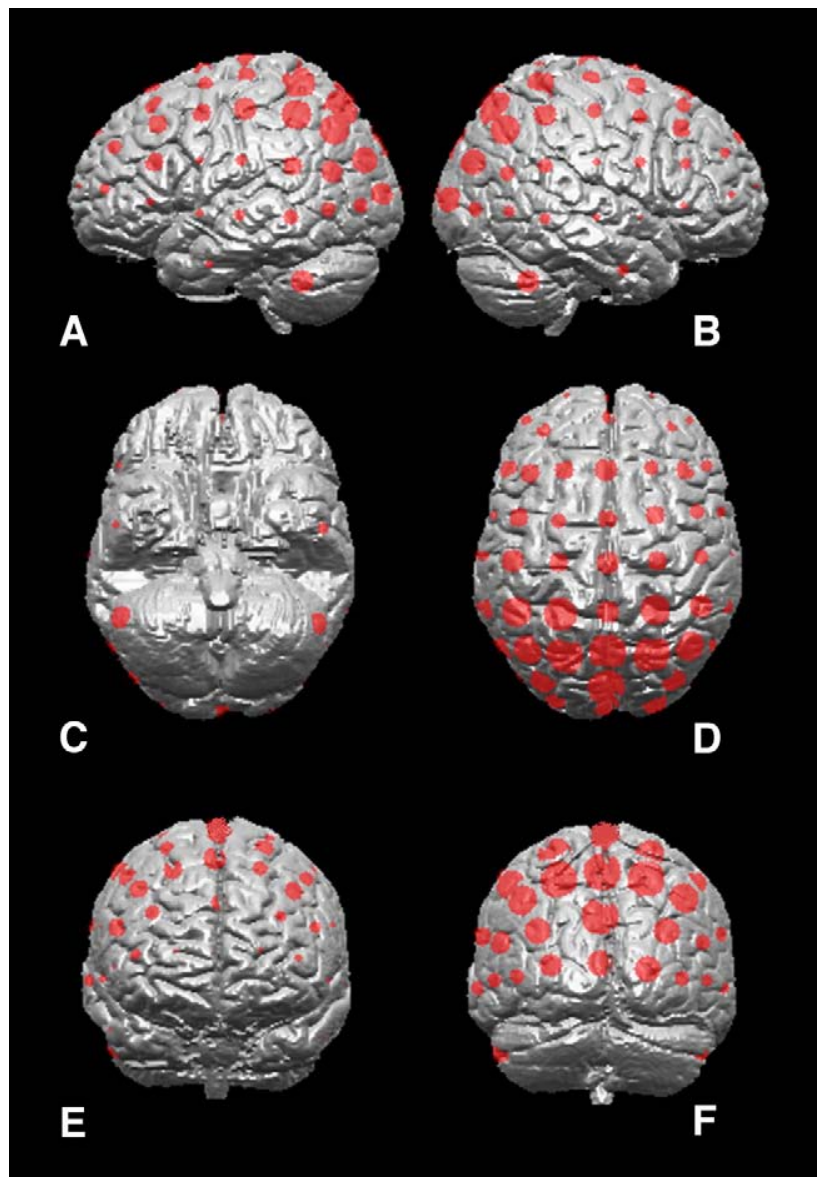

Fig. 5. Average cortical projection points for 10-10 standard positions in 16 subjects. Red circles centred on each mean cortical projection point have a diameter proportional to the standard deviation in 3D. (A) Left temporal view, (B) right temporal view, (C) bottom view, (D) top view, (E) front view, (F) bottom view.

\section{Statistical analysis}

Macro-anatomical variations of each sensor are presented in Table 2. In our study, we observed that a small dispersion of cortical projections was present in the pre-frontal and occipital regions. More than $80 \%$ of the sensors were always on the same BA. In contrast, a large dispersion was observed around the sylvian fissure and central region. In these cases, several BAs (three, four and sometimes five) were targeted by projection of the same sensor in different subjects. In some cases this variability did not allow us to establish a unique predominant BA.

\section{Discussion}

The aim of this study was to present a 3D anatomical atlas which completes our knowledge of the current anatomical correlations with 10-20 EEG sensors. We examined the cranio-cerebral correlations and probabilistically expressed the locations of $10-10$ standard positions and their cortical projection points in standard stereotactic space for brain imaging studies with anatomical considerations.

Conceptually, our approach is an extension of the work of Okamoto et al. (2004), who projected the 10-20 standard positions onto Brodmann's atlas (Brodmann, 1909, 1912) and thus expressed their cortical projection points in the 3D Talairach atlas (Talairach and Tournoux, 1988). Considering anatomical points (i.e. nasion and preauriculars) to position surface EEG sensors in the fiducial system, we transformed these landmarks into the Talairach system. These two independent mathematical transformations of coordinates do not normalize brain images and conserve anatomical features of each patient, which is fundamental for such a macro-anatomical study. The Talairach atlas (Talairach and Tournoux, 1988) is the most commonly used system for presenting coordinates in neuroimaging studies and is used in both BrainMap (Laird et al., 2005) and Talairach Daemon (Lancaster et al., 2000). One of the most important drawbacks is the absence of an actual Talairach-brain image. Several studies (Lancaster et al., 2007; Lacadie et al., 2008) have developed new systems to provide more accurate Talairach coordinates for neuroimaging and to correct the bias between MNI and Talairach coordinates. However, the transition from Talairach to MNI stereotactic coordinate systems (reviewed in Brett et al., 2002) is still in process. It is possible, of course that this transition may never be fully completed and that researchers will ultimately opt for the coexistence of the two systems (Jurcak et al., 2007).

In our study, we used a projection method that we integrated into the ALLES algorithm (Koessler et al., 2008). Our projection method differs from others described in the literature (Homan et al., 1987; Okamoto and Dan, 2005) which project EEG sensors perpendicularly to the cortical surface or to the closest point on the cortical surface.

Our projection method is similar to the algorithm described by Okamoto and Dan (2005). The main difference is that our approach uses regions of interest defined by spheres to obtain cortical projections whereas Okamoto's approach uses planes defined by a rigid number of points. Consequently, our cortical projections depend on barycentres of regions of interest while Okamoto's algorithm depends on closest points of the convex hull surface and cortical surface. Our approach with barycentre calculation always gives a single solution by taking into account the cerebral volumes concerned by the surface sensors. The advantage of our solution is that it overcomes the issue of multiple solutions encountered with the closest cortical projection method. After tests, we can confirm that variations in the diameter of the imaginary sphere do not cause significant displacements of cortical projections and consequently do not change the anatomical structure beneath the EEG sensor.

The anatomical correlation of the $10-10$ system is original because previous studies have presented only correlations within the 10-20 system. With the increased number of EEG sensors we have improved 

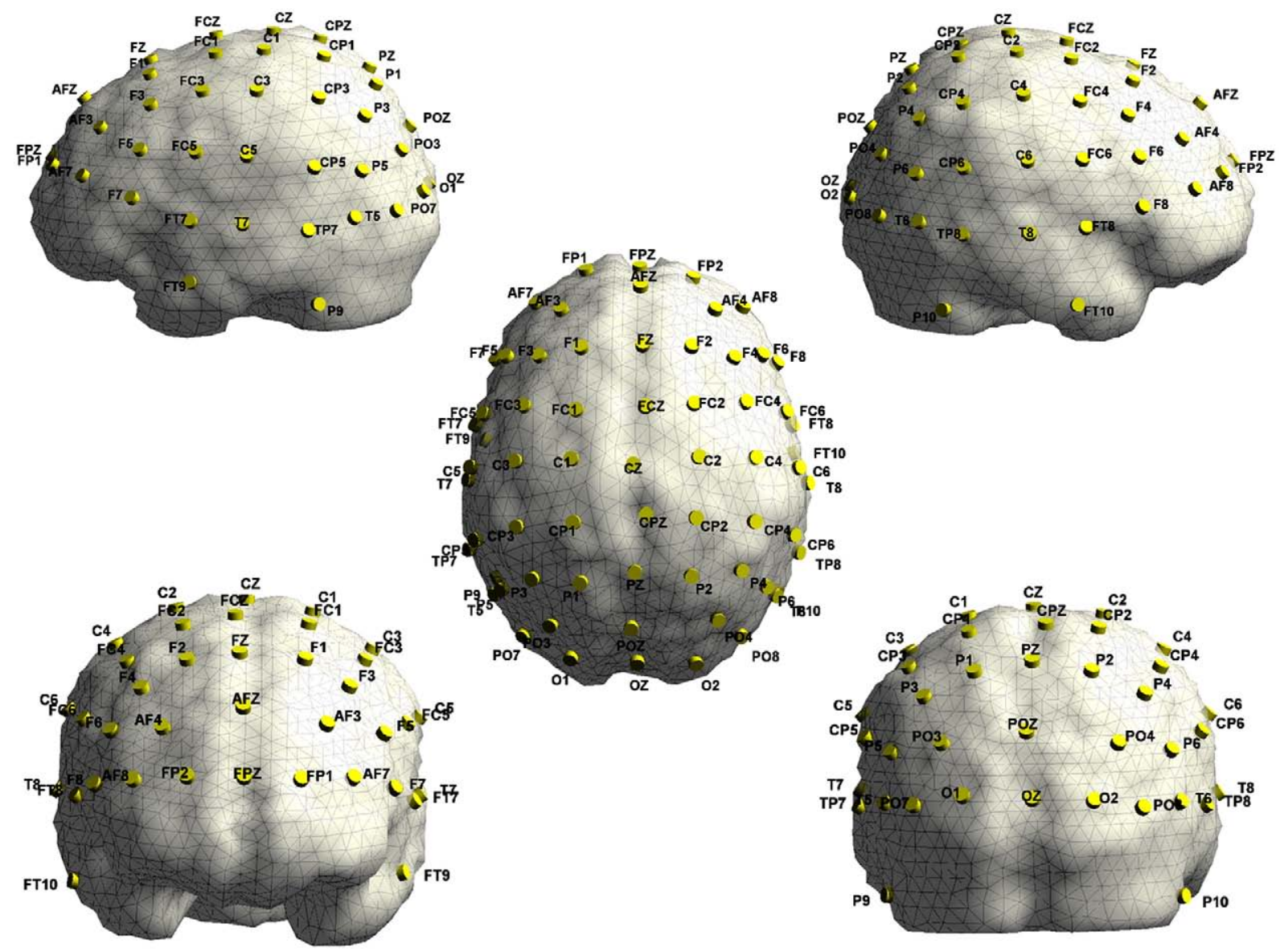

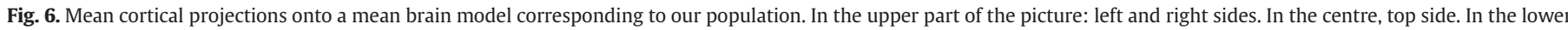
part of the picture, front and back sides.

the precision of cortical correlations, notably in regions which present several different anatomo-functional structures (central sulcus with BA 6, 4 and 123, and the sylvian fissure with BA 40, 41, 44/45 and 38). More precise macro-anatomical and cytoarchitectonic definitions would help further neurophysiologists in the field of surface cortical imaging. This automated projection technique is well adapted for NIRS and TMS because the source is estimated only on the lateral cortical surface.

In this study, we observed that the mean cortical projections of F1, $\mathrm{Fz}$ and F2 are on the superior frontal gyrus whereas F5, F3, F4 and F6 are on the middle frontal gyrus. In the same way, mean cortical projections of $\mathrm{C} 1$ and $\mathrm{C} 2$ are on the pre-central gyrus whereas $\mathrm{C} 5, \mathrm{C} 3$, C4 and C6 are on the post-central gyrus.

In our study, 10-20 cranio-cerebral correlations are in accordance with those described in the literature (Lagerlund et al., 1993; Okamoto and Dan, 2005). Some differences concerning BA could be explained by variability in the limits between some BAs such as $40,41,44$ and 45 . Concerning the variability of the cortical projections, we demonstrated in our population that several sensors were always projected in the same area (Fp1, Fp2, $\mathrm{O} 1$ and $\mathrm{O} 2$ ) whereas others could be projected on several different areas (C6 and FC6). The most important variations in the $y$ axis are observed in the median part (FC, C and CP) of the brain. In the $z$ axis, the most important variations are visible in the posterior regions ( $\mathrm{P}, \mathrm{PO}$ and $\mathrm{O}$ ). The manual positioning of the EEG sensors explains much of the variability in cortical projections. A small displacement of a sensor can induce a projection on two different cortical structures. This problem can be highlighted for sensors situated in the central region where the anterior and posterior banks of the central sulcus are separated by only a few millimetres. This shows that results obtained from manual positioning are slightly different from virtual 10-20 measurements on MR images as described by Jurcak et al. (2005). This experimental variability justifies the development of a realistic cranio-cerebral database for clinical purposes. Identification of external landmarks can be very difficult during EEG sensor positioning, in particular the nasion and inion. Some of the cortical variations can be explained by these difficulties. Definitions of the 10-10 system and its derivatives remain ambiguous and this reduces the potential accuracy of these systems. Ideally, to enhance accuracy, the current definitions should be revised to give more detailed methods for setting landmarks. However, in practice, it takes time to realize such standardization. In our laboratory, only Oostenveld's configuration of EEG sensors is currently used and has been studied, but several others exist and present spatial variability which is outside the scope of the present study (Jurcak et al., 2007).

Moreover, cortical variability can be partly explained by the independent anatomical development of the skull and brain. Consequently, it is possible to observe different cortical coordinates beneath the same cranial landmark in a population.

Another possible source of variation is localization of the EEG sensors using MR images. Small geometric distortions can induce cortical projection errors. Visual inspection of MR images by physicians was carried out in order to avoid this kind of error. Finally, brain segmentation can lead to cortical projection errors because in a few patients $(n=3)$ it was difficult to optimize the grey scale threshold and obtain a realistic head model, notably in the vertex area.

Knowledge of cortical projections of EEG sensors may have several applications in brain imaging such as NIRS and TMS. In spectroscopy, a signal from the surface (i.e. light recorded by detectors) comes from superficial brain sources. In TMS, it is well recognized that precise 
Table 2

Macro-anatomical and cytoarchitectonic variabilities of cortical projections in the 10-10 system

\begin{tabular}{|c|c|c|c|c|}
\hline Labels & Macro-anatomical variabilities & Main macro-anatomical structures & Main BA & Cytoarchitectonic (Brodmann) variabilities \\
\hline$\overline{\text { Fp1 }}$ & GFS (65\%) GFM (35\%) & Superior frontal G & 10 & $10(100 \%)$ \\
\hline Fpz & GFd (66\%) SI (17\%) GFM (17\%) & Medialis frontal G & 10 & $10(100 \%)$ \\
\hline Fp2 & GFS (75\%) GFM (25\%) & Superior frontal G & 10 & $10(100 \%)$ \\
\hline AF7 & GFM $(100 \%)$ & Middle frontal G & 10 & $10(75 \%), 46(25 \%)$ \\
\hline AF3 & GFS (56\%) GFM (44\%) & Superior frontal G & 9 & 9 (75\%), 10 (19\%), 8 (6\%) \\
\hline $\mathrm{AFz}$ & GFS (75\%) GFd (19\%) SI (6\%) & Superior frontal G & 9 & 9 (62,5\%), $6(12,5 \%), 8(19 \%), 10(6 \%)$ \\
\hline AF4 & GFS (75\%) GFM (25\%) & Superior frontal G & 9 & 9 (69\%), $10(25 \%), 8(6 \%)$ \\
\hline AF8 & GFM (81\%) GFS (13\%) GFI (6\%) & Middle frontal G & 10 & 10 (81\%), 49 (19\%) \\
\hline F7 & GFI $(100 \%)$ & Inferior frontal G & 45 & 45 (56\%), 47 (38\%), $46(6 \%)$ \\
\hline F5 & GFM (88\%) GTS (6\%) GFI (6\%) & Middle frontal G & 46 & 46 (50\%), $9(38 \%), 45(6 \%), 22(6 \%)$ \\
\hline F3 & GM (75\%) GFS (25\%) & Middle frontal G & 8 & 8 (75\%), 6 (19\%), 46 (6\%) \\
\hline $\mathrm{F} 1$ & GFS $(88 \%)$ GFM ( $12 \%)$ & Superior frontal G & 6 & $6(63 \%), 8(31 \%), 9(6 \%)$ \\
\hline $\mathrm{Fz}$ & GFS (81\%) SI (19\%) & Superior frontal G & 6 & $6(81,5 \%), 8(12,5 \%), 9(6 \%)$ \\
\hline F2 & GFS (75\%) GFM (25\%) & Superior frontal G & 6 & $6(69 \%), 8(31 \%)$ \\
\hline F4 & GFM (63\%) GFS (31\%) GPREC (6\%) & Middle frontal G & 8 & 8 (69\%), 6 (6\%), 9 (25\%) \\
\hline F6 & GFM (75\%) GFI (25\%) & Middle frontal G & 9 & $9(43,5 \%), 46(37,5 \%), 45(19 \%)$ \\
\hline F8 & GFI (88\%) GFM (12\%) & Middle frontal G & $45 / 47$ & $45(37,5 \%), 47(37,5 \%), 46(25 \%)$ \\
\hline FT7 & GTS (82\%) GTM (12\%) GFI (6\%) & Superior temporal & 22 & $22(75,5 \%), 21(12,5 \%), 38(6 \%), 44(6 \%)$ \\
\hline FC5 & GPREC $(63 \%)$ GFI (37\%) & Precentral G & 6 & $6(63 \%), 9(25 \%), 44(6 \%), 45(6 \%)$ \\
\hline FC3 & GFM (63\%) GPREC (37\%) & Middle frontal G & 6 & $6(75 \%), 4(12,5 \%), 8(12,5 \%)$ \\
\hline FC1 & GFS (88\%) GFM (12\%) & Superior frontal G & 6 & $6(100 \%)$ \\
\hline $\mathrm{FCz}$ & SI (50\%) GFS (31\%) GFM (19\%) & Interhemispheric sulcus & 6 & $6(100 \%)$ \\
\hline $\mathrm{FC} 2$ & GFS (56\%) GFd (38\%) GPREC (6\%) & Superior frontal G & 6 & $6(100 \%)$ \\
\hline FC4 & GFM (75\%) GPREC (19\%) GPSTC (6\%) & Middle frontal G & 6 & 6 (82\%), 123 (6\%), 8 (6\%), 9 (6\%) \\
\hline FC6 & GPREC (63\%) GFI (25\%) GFM (6\%) GPSTC (6\%) & Precentral G & 6 & $6(56,5 \%), 9(19,5 \%), 43(6 \%), 44(6 \%), 45(6 \%), 8(6 \%)$ \\
\hline FT8 & GTS $(81 \%)$ GTM (13\%) GPREC (6\%) & Superior temporal G & 22 & $22(75 \%), 21(13 \%), 38(6 \%), 44(6 \%)$ \\
\hline $\mathrm{T} 7$ & GTM (69\%) GTS (19\%) GPSTC (12\%) & Middle temporal G & 21 & $21(81,5 \%), 22(12,5 \%), 43(6 \%)$ \\
\hline C5 & GPSTC (69\%) LPI (25\%) GPREC (6\%) & Postcentral G & 123 & $123(44 \%), 40(37,5 \%), 43(12,5 \%), 6(6 \%)$ \\
\hline C3 & GPSTC (69\%) GPREC (19\%) LPI (12\%) & Postcentral G & 21 & $21(62,5 \%), 22(25 \%), 20(6,5), 42(6 \%)$ \\
\hline $\mathrm{C} 1$ & GPREC (63\%) GPSTC (25\%) GFS (13\%) & Precentral G & $4 / 6$ & $4(37,5 \%), 6(37,5 \%), 123(25 \%)$ \\
\hline $\mathrm{Cz}$ & SI $(81 \%)$ GFS (6\%) GFM (6\%) LPARAC (6\%) & Interhemispheric scissure & 4 & $4(62,5 \%), 6(37,5 \%)$ \\
\hline $\mathrm{C} 2$ & GPREC (63\%) GPSTC (25\%) GFS (13\%) & Precentral G & 123 & $123(56,5 \%), 40$ (25,5\%), 4 (12,5\%), $6(6 \%)$ \\
\hline C4 & GPSTC ( $81 \%)$ GPREC (13\%) LPI (6\%) & Postcentral G & 123 & $123(81,5 \%), 6(12,5), 40(6 \%)$ \\
\hline C6 & GPSTC (50\%) LPI (25\%) GPREC (25\%) & Postcentral G & $123 / 40$ & $\begin{array}{l}123(25 \%), 40(25 \%), 4(12,5 \%), 6(12,5 \%), \\
43(12,5 \%), 2(12,5 \%)\end{array}$ \\
\hline T8 & GTM (56\%) GTS (38\%) GTI (6\%) & Middle temporal G & 4 & 4 (50\%), 123 (25\%), 6 (25\%) \\
\hline TP7 & GTM $(82 \%)$ GTI (12\%) GTS (6\%) & Middle temporal G & 21 & $21(50 \%), 37$ (25\%), 22 (19\%), 20 (6\%) \\
\hline CP5 & GTS (5\%) GSM (24\%) GTM (13\%) LPI (13\%) & Superior temporal G & 22 & 22 (44\%), 40 (37,5\%), 39 (12,5\%), 21 (6\%) \\
\hline CP3 & LPI (75\%) GPSTC ( $13 \%)$ LPS (6\%) GA (6\%) & Inferior parietal L & 40 & 40 (82\%), $123(6 \%), 5(6 \%), 39(6 \%)$ \\
\hline CP1 & LPS (50\%) GPSTC (50\%) & Postcentral G-Superior parietal L & 7 & $7(62,5 \%), 5$ (31,5\%), $123(6)$ \\
\hline $\mathrm{CPz}$ & GPSTC $(44 \%)$ SI $(38 \%)$ PC (18\%) & Postcentral G & 7 & 7 (56\%), 5 (19\%), $123(12,5 \%), 4(12,5 \%)$ \\
\hline $\mathrm{CP} 2$ & GPSTC (56\%) LPS (44\%) & Postcentral G & 5 & $5(62,5 \%, 7(25 \%), 123(12,5 \%)$ \\
\hline $\mathrm{CP} 4$ & LPI (88\%) GPSTC (12\%) & Inferior parietal $\mathrm{L}$ & 40 & $40(77,5 \%), 123(12,5 \%)$ \\
\hline CP6 & GSM (38\%) GTS (38\%) LPI (24\%) & Superior temporal G-GSM & 40 & $40(62,5 \% 0), 22(37,5 \%)$ \\
\hline TP8 & GTM (56\%) GTI (31\%) GTS (13\%) & Middle temporal G & 21 & $21(62,5 \%), 22(12,5 \%), 20(12,5 \%), 37(12,5 \%)$ \\
\hline P7 & GOM (38\%) GTM (25\%) GTI (25\%) GTS (6\%) GF (6\%) & Middle occipital G & 37 & 37 (44\%), 19 (38\%), 39 (18\%) \\
\hline P5 & $\begin{array}{l}\text { GTM (56\%) GA (13\%) GOM (13\%) GSM (6\%) } \\
\text { GTS (6\%) LPI (6\%) }\end{array}$ & Middle temporal G & 39 & 39 (62,5\%), 19 (19\%), 37 (12,5\%), 40 (6\%) \\
\hline P3 & LPI (38\%) PC (25\%) GA (19\%) LPS (12\%) GTM (6\%) & Inferior parietal L & 39 & 39 (37,5\%), 7 (25\%), 19 (25\%), $40(12,5 \%)$ \\
\hline P1 & PC (50\%) LPS (44\%) GPSTC (6\%) & Precuneus & 7 & $7(87,5 \%), 19(12,5 \%)$ \\
\hline $\mathrm{Pz}$ & PC (62\%) LPS (19\%) SI (19\%) & Precuneus & 7 & 7 (88\%), 5 (6\%), 19 (6\%) \\
\hline P2 & PC (63\%) LPS (31\%) GPSTC (6\%) & Precuneus & 7 & 7 (81,5\%), 19 (12,5\%), $5(6 \%)$ \\
\hline P4 & LPI $(31 \%)$ GA (31\%) LPS (19\%) PC (13\%) GOS (6\%) & Inferior parietal L & 39 & 39 (31\%), 7 (25\%), 40 (25\%), $19(19 \%)$ \\
\hline P6 & GTM (69\%) GA (13\%) LPI (6\%) GTS (6\%) GOM (6\%) & Middle temporal G & 39 & $39(75,5 \%), 19(12,5 \%), 40(6 \%), 37(6 \%)$ \\
\hline P8 & GTI (44\%) GOM (31\%) GTM (19\%) GTS (6\%) & Inferior temporal G & 19 & 19 (56\%), $37(19 \%), 20(12,5), 39(12,5 \%)$ \\
\hline PO7 & GOM (63\%) GOI (31\%) GA (6\%) & Middle occipital G & 19 & $19(62,5 \%), 18(31 \%), 39(6,5 \%)$ \\
\hline PO3 & GOM $(50 \%)$ PC (18\%) C (13\%) GOS (13\%) GTM (6\%) & Middle occipital G & 19 & $19(75,5 \%), 7(6 \%), 39(6 \%), 18(12,5 \%)$ \\
\hline $\mathrm{POz}$ & C (69\%) PC (25\%) LPS (6\%) & Cuneus & 19 & 19 (56\%), 18 (25\%), 7 (19\%) \\
\hline PO4 & $\begin{array}{l}\text { GOM (38\%) GOS (19\%) GTM (19\%) C (12\%) LPS (6\%) } \\
\text { PC (6\%) }\end{array}$ & Middle occipital G & 19 & 19 (69\%), 39 (12,5\%), 18 (12,5\%), 7 (6\%) \\
\hline PO8 & GOM (44\%) GOI (44\%) GOS (6\%) GTM (6\%) & Middle occipital G & 19 & 19 (69\%), $18(31 \%)$ \\
\hline 01 & GOM (38\%) C (19\%) GL (19\%) GOI (19\%) PC (5\%) & Middle occipital G & 18 & 18 (81\%), 19 (19\%) \\
\hline $\mathrm{Oz}$ & C (98\%) GL (5\%) GOM (6\%) & Cuneus & 18 & 18 (62,5), 17 (31\%), $19(6,5 \%)$ \\
\hline $\mathrm{O} 2$ & C (38\%) GOM (31\%) GL (25\%) GOI (6\%) & Cuneus & 18 & $18(81 \%), 19(19 \%)$ \\
\hline
\end{tabular}

identification of the cortical target enhances the quality of stimulation. In contrast, in most cases, this tool cannot be used for EEG signal localization because of the variable depth and orientation of brain generators (Gloor, 1985; Ebersole, 2003). Moreover, it is now well known that the EEG signal comes from a large cortical area (Cosandier-Rimélé et al., 2007; Tao et al., 2007) and thus EEG source localization cannot be inferred from cranio-cerebral correlations.
However, in particular situations such as focal and superficial dysplasia, identification of cortical structures beneath EEG sensors can be helpful for spatial localization. In this kind of pathology, dysplasia produces typical surface EEG signals (Gambardella et al., 1996). If the surface EEG signal is very focal and concerns only few sensors, the technique will be useful for marking the boundary of the dysplastic area. At last, in the case of superficial and radial dipolar sources, the 
combined use of cranio-cerebral correlations and topography can give a good indication of the location of the cerebral source. Finally, the use of automated cortical projections combined with neuronavigation could be compared to electrocorticography to guide epilepsy surgery (Morino et al., 2004).

\section{Acknowledgments}

We thank Mr. Christian-G. Bénar for examination of the manuscript and for his helpful suggestions. We also gratefully acknowledge the participation of the individuals involved in this study. This study was supported by the company T.E.A of Nancy (France) and the Regional Council of Lorraine (France).

\section{Appendix A}

Canonical MRI coordinates of the nasion $(N)$, left ear (LE) and right ear $(\mathrm{RE})$ defined the origin $(O)$ which can be computed as:

$O=\frac{\mathrm{LE}+(\mathrm{LE}-\mathrm{RE})[(N-\mathrm{LE})(\mathrm{LE}-\mathrm{RE})]}{\|\mathrm{LE}-\mathrm{RE}\| 2}$.

\section{ASA software then defined unity vectors for new axes:}

$e_{x}=\frac{(N-O)}{\|N-O\|} \quad e_{y}=\frac{(\mathrm{LE}-O)}{\|L E-O\|} e_{z}=e_{x} e_{y}$.

A rotation matrix was then calculated as: $R=\left(e_{\mathrm{x}} e_{y} e_{z}\right)^{T}$.

Finally, in order to obtain the fiducial system, shift and rotation were applied:

$P_{\text {fiducial }}=R\left(P_{\text {canonical }}-0\right)$.

To transform the fiducial system to the Talairach system, ASA used these coordinates:

\begin{tabular}{lrcr}
\hline & $x(\mathrm{~mm})$ & $y(\mathrm{~mm})$ & $z(\mathrm{~mm})$ \\
\hline PC & -23 & 0 & 0 \\
AP & 70 & 0 & 0 \\
PP & -102 & 0 & 0 \\
IP & 0 & 0 & -42 \\
SP & 0 & 0 & 74 \\
RP & 0 & -68 & 0 \\
LP & 0 & 68 & 0 \\
\hline
\end{tabular}

Transformations from the fiducial system to the Talairach system were then calculated:

$$
\begin{aligned}
& P_{\text {Fiducial }}=(x, y, z) \\
& P_{\text {Talairach }}=\left(x_{\mathrm{t}}, y_{\mathrm{t}}, z_{\mathrm{t}}\right) \\
& \text { if }(x<0) x_{\mathrm{t}}=\left(x 1_{\mathrm{T}} / x 1\right) x / / \text { anterior to } O \\
& \text { if }(x \geq 0) x_{\mathrm{t}}=\left(x 2_{\mathrm{T}} / x 2\right) x / / \text { posterior to } O \\
& \text { if }(y<0) y_{\mathrm{t}}=\left(y 1_{\mathrm{T}} / y 1\right) y / / \text { right hemisphere } \\
& \text { if }(y \geq 0) y_{\mathrm{t}}=\left(y 2_{\mathrm{T}} / y 2\right) y / / \text { left hemisphere } \\
& \text { if }(z<0) z_{\mathrm{t}}=\left(z 1_{\mathrm{T}} / z 1\right) z / / \text { inferior to } O \\
& \text { if }(z \geq 0) z_{\mathrm{t}}=\left(z 2_{\mathrm{T}} / z 2\right) z / / \text { superior to } O
\end{aligned}
$$

\section{References}

Blume, W.T., Buza, R.C., Okazaki, H., 1974. Anatomical correlates of the ten-twenty electrode placement system in infants. Electroencephalogr. Clin. Neurophysiol. 36, 303-307.

Brett, M., Johnsrude, I.S., Owen, A.M., 2002. The problem of functional localization in the human brain. Nat. Rev. Neurosci. 3, 243-249.

Brodmann, K., 1909. Vergleichende Lokalisationslehre der Grosshirnrinde in ihren Prinzipien dargestellt auf Grund des Zellenbaues. J.A. Barth, Leipzig.

Brodmann, K., 1912. Neue Ergebnisse über die vergleichende histologische Lokalisation der Grosshirnrinde. Anat. Anz. 41, 157-216.
Cosandier-Rimélé, D., Badier, J.-M., Chauvel, P., Wendling, F., 2007. A physiologically plausible spatiotemporal model for EEG signals recorded with intracerebral electrodes in human partial epilepsy. IEEE Trans. Biomed. Eng. 54 (3), 380-388.

Ebersole, J.S., 2003. Cortical generators and EEG voltage fields. In: Ebersole, JS, Pedley, TA (Eds.), Current practice of clinical electroencephalography. Lippincott Williams and Wilkins, Philadelphia, pp. 12-31.

Gambardella, A., Palmini, A., Andermann, F., Dubeau, F., Da Costa, J.C., Quesney, L.F., Andermann, E., Olivier, A., 1996. Usefulness of focal rhythmic discharges on scalp EEG of patients with focal cortical dysplasia and intractable epilepsy. Electroencephalogr. Clin. Neurophysiol. 98 (4), 243-249.

Gloor, P., 1985. Neuronal generators and the problem of localization in electroencephalography: application of volume conductor theory to electroencephalography. J. Clin. Neurophysiol. 2 (4), 327-354.

Homan, R.W., Herman, J., Purdy, P., 1987. Cerebral location of international 10-20 system electrode placement. Electroencephalogr. Clin. Neurophysiol. 66, 376-382.

Jack, C.R., Marsh, W.R., Hirschorn, K.A., Shabrough, F.W., Cascino, G.D., Karwoski, R.A., Robb, R.A., 1990. EEG scalp electrode projection onto 3-dimensional surface rendered images of the brain. Radiology 176, 413-418.

Jasper, H.H., 1958. The ten-twenty electrode system of the international federation. Electroencephalogr. Clin. Neurophysiol. 10, 367-380.

Jurcak, V., Okamoto, M., Singh, A., Dan, I., 2005. Virtual 10-20 measurement on MR images for inter-modal linking of transcranial and tomographic neuroimaging methods. NeuroImage 26 (4), 1184-1192.

Jurcak, V., Tsuzuki, D., Dan, I., 2007.10/20,10/10, and 10/5 systems revisited: their validity as relative head-surface-based positioning systems. Neurolmage 34 (4), 1600-1611.

Klem, G.H., Lüders, H.O., Jasper, H.H., Elger, C., 1999. The ten-twenty electrode system of the International Federation. International Federation of Clinical Neurophysiology. Electroencephalogr. Clin. Neurophysiol. Suppl. 52, 3-6.

Koessler, L., Benhadid, A., Maillard, L., Vignal, J.P., Vespignani, H., Braun, M., 2008. Automatic localization of new scalp-recorded EEG sensors in MRI volume. Neurolmage 41 (3), 914-923.

Lacadie, C.M., Fulbright, R.K., Rajeevan, N., Constable, R.T., Papademetris, X., 2008. More accurate Talairach coordinates for neuroimaging using non-linear registration. NeuroImage 42 (2), 717-725.

Lagerlund, T.D., Sharbrough, F.W., Jack Jr., C.R., Erickson, B.J., Strelow, D.C., Cicora, K.M., Busacker, N.E., 1993. Determination of 10-20 system electrode locations using magnetic resonance image scanning with markers. Electroencephalogr. Clin. Neurophysiol. 86, 7-14.

Laird, A.R., Lancaster, J.L., Fox, P.T., 2005. BrainMap: the social evolution of a functional neuroimaging database. Neuroinformatics 3, 65-78.

Lancaster, J.L., Woldorff, M.G., Parsons, L.M., Liotti, M., Freitas, C.S., Rainey, L., Kochunov P.V., Nickerson, D., Mikiten, S.A., Fox, P.T., 2000. Automated Talairach atlas labels for functional brain mapping. Hum. Brain Map. 10, 120-131.

Lancaster, J., Tordesillas-Gutirrez, D., Martinez, M., Salinas, F., Evans, A., Zilles, K. Mazziotta, J., Fox, P., 2007. Bias between MNI and Talairach coordinates analyzed using the ICBM-152 brain template. Hum. Brain Map. 28 (11), 1194-1205.

Lantz, G., Grave de Peralta, R., Spinelli, L., Seeck, M., Michel, C.M., 2003. Epileptic source localization with high density EEG: how many electrodes are needed? Clin. Neurophysiol. 114, 63-69.

Michel, C.M., Murray, M.M., Lantz, G., Gonzalez, S., Spinelli, L., Grave de Peralta, R., 2004 EEG source imaging. Clin. Neurophysiol. 115 (10), 2195-2222.

Morino, M., Ishibashi, K., Hara, M., 2004. Surgical treatment of temporal lobe epilepsy associated with subcortical ectopic gray matter under the guidance of intraoperative electrocorticography. Seizure 13 (7), 470-474.

Morris, H.H., Lüders, H., Lesser, R.P., Dinner, D.S., Klem, G.H., 1986. The value of closely spaced scalp electrodes in the localization of epileptiform foci: a study of 26 patients with complex partial seizures. Electroencephalogr. Clin. Neurophysiol. 63, 107-111.

Myslobodsky, M.S., Bar-Ziv, J., 1989. Locations of occipital EEG electrodes verified by computed tomography. Electroencephalogr. Clin. Neurophysiol. 72, 362-366.

Myslobodsky, M.S., Coppola, T., Bar-Ziv, J., Weinberger, D.R., 1990. Adequacy of the international 10-20 electrode system for computed neurophysiologic topography. J. Clin. Neurophysiol. 7, 507-518.

Nuwer, M.R., Comi, G., Emerson, R., Fuglsang-Frederiksen, A., Guerit, J.M., Hinrichs, H., Ikeda, A., Luccas, F.J., Rappelsburger, P., 1998. IFCN standards for digital recording of clinical EEG. International Federation of Clinical Neurophysiology. Electroencephalogr. Clin. Neurophysiol. 106, 259-261.

Okamoto, M., Dan, I., 2005. Automated cortical projection of head-surface locations for transcranial functional brain mapping. NeuroImage 26, 18-28.

Okamoto, M., Dan, H., Sakamoto, K., Takeo, K., Shimizu, K., Kohno, S., Oda, I., Isobe, S., Suzuki, T., Kohyama, K., Dan, I., 2004. Three dimensional probabilistic anatomical cranio-cerebral correlation via the international 10-20 system oriented for transcranial functional brain mapping. Neurolmage 21, 99-111.

Oostenveld, R., Praamstra, P., 2001. The five percent electrode system for highresolution EEG and ERP measurements. Clin. Neurophysiol. 112, 713-719.

Steinmetz, H., Furst, G., Meyer, B.U., 1989. Craniocerebral topography within the international 10-20 system. Electroencephalogr. Clin. Neurophysiol. 72, 499-506.

Talairach, J., Tournoux, P., 1988. Co-planar stereotaxic atlas of the human brain. Thieme, New York.

Tao, J., Baldwin, M., Hawes-Ebersole, S., Ebersole, J., 2007. Cortical substrates of scalp EEG epileptiform discharges. J. Clin. Neurophysiol. 24, 96-100.

Towle, V.L., Bolanos, J., Suarez, D., Tan, K., Grzeszczuk, R., Levin, D.N., Cakmur, R., Frank, S.A., Spire, J.P., 1993. The spatial location of EEG electrodes: locating the bestfitting sphere relative to cortical anatomy. Electroencephalogr. Clin. Neurophysiol. $86,1-6$. 